\section{Novel NLRP12 variant presenting with familial cold autoimmunity syndrome phenotype}

We read with interest the paper by Ter Haar et al describing 187 patients with undefined autoinflammatory syndromes, some of whom had variants of unknown significance (VOUS) in the known genes. ${ }^{1}$ Patients with genetic mutations had a higher frequency of family history of similar disorder, suggesting that these variants may have some role to play.

We report a 4-year-old girl who presented with familial cold autoinflammatory syndrome-2 (FCAS2) phenotype and a novel mutation in leucine-rich repeat (LRR) domain of the nucleotide oligomerization domain (NOD)-like receptor protein 12 (NLRP12). ${ }^{23}$

This child had episodic fever each lasting a fortnight and occurring once or twice a month. It was associated with recurrent, watery, non-infective diarrhoea since birth. Stools were occasionally admixed with blood. Colonoscopy was normal but biopsy showed cryptitis. She had several infections in childhood: one probable meningitis, one pneumonia and two episodes of subcutaneous abscesses. She had developed additive arthritis of large joints (knees, shoulders and elbows). Arthritis had a relapsing and remitting course. At 1.5 years of age, she was noticed to have sensorineural hearing loss (SNHL). She was borne out of a second-degree consanguineous marriage, her mother had a history of stillbirth in the previous pregnancy.

The child's height and weight were below the fifth centile. She had bilateral non-tender cervical lymphadenopathy and mild hepatosplenomegaly. Labs revealed neutrophilic leucocytosis, thrombocytosis and elevated acute phase reactants during febrile spells, with moderate microcytic hypochromic anaemia. Numerous cultures of blood, urine and stool were sterile.
The symptom complex of periodic fever, arthritis, sterile colitis and SNHL was consistent with cold associated autoinflammatory syndromes, although the attacks were not precipitated by cold in this child. Recurrent infections brought in the possibility of an associated underlying primary immunodeficiency (PID), which has been reported with NLRP12 defects.

Though workup for PID was negative (normal immunoglobulins, lymphocyte subsets, dihydrorhodamine assay for neutrophil oxidative burst, and baseline and lipopolysaccaride-stimulated production of tumour necrosis factor in whole blood cultures). Whole exome sequencing revealed a novel mutation in the exon 9 of the NLRP12 gene (c.54299276T>C:r.2935a>g:p. Ser979Gly) on chromosome 19. Sanger sequencing confirmed the presence of this homozygous variant in proband and parents were found to be heterozygous for this mutation (figure 1).

On follow-up, arthritis responded to naproxen but the fever persisted. Due to non-availability of interleukin-1 inhibitor, she was treated with prednisolone, with which she has good response. Meanwhile, her mother had another child for whom prenatal diagnosis showed no NLRP12 mutation.

The p.S979G mutation has not been reported so far. In silico prediction tools, MutationTaster and PolyPhen-2, suggested that this variant is probably damaging to the protein function. This missense variant alters a conserved residue in the protein function. In silico studies on the effect of this mutation to protein structure were inconclusive. This mutation in exon 9 possibly interferes with Pathogen Associated Molecular Pattern recognition leading to both autoimmunity and susceptibility to infection. Overall, p.F402L accounts for more than half of the cases of FCAS2. ${ }^{4}$ p.H304Y has been reported in association with Common Variable Immunodeficiency. Most of the variants have been reported in the NOD domain.

\section{Proband}

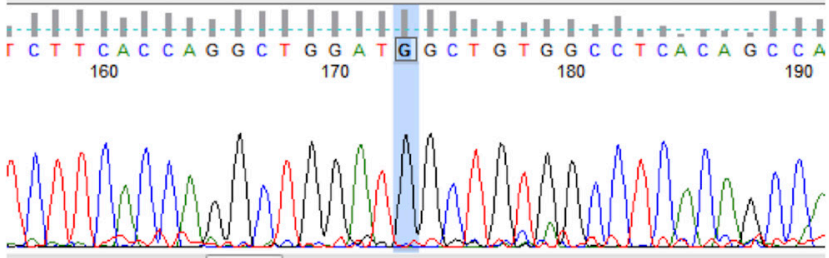

Mother

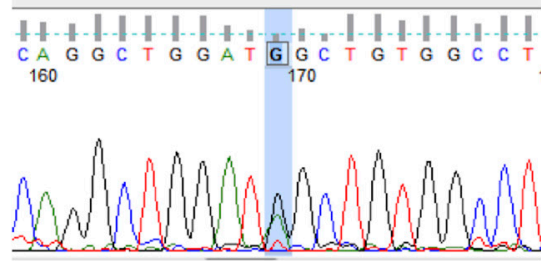

tomo sapiens NLR family pyrin domain containing 12 (NLRP12), RefSeqGene on chromosome 19 iequence ID: refING_008651.1 Length: 37811 Number of Matches: 1
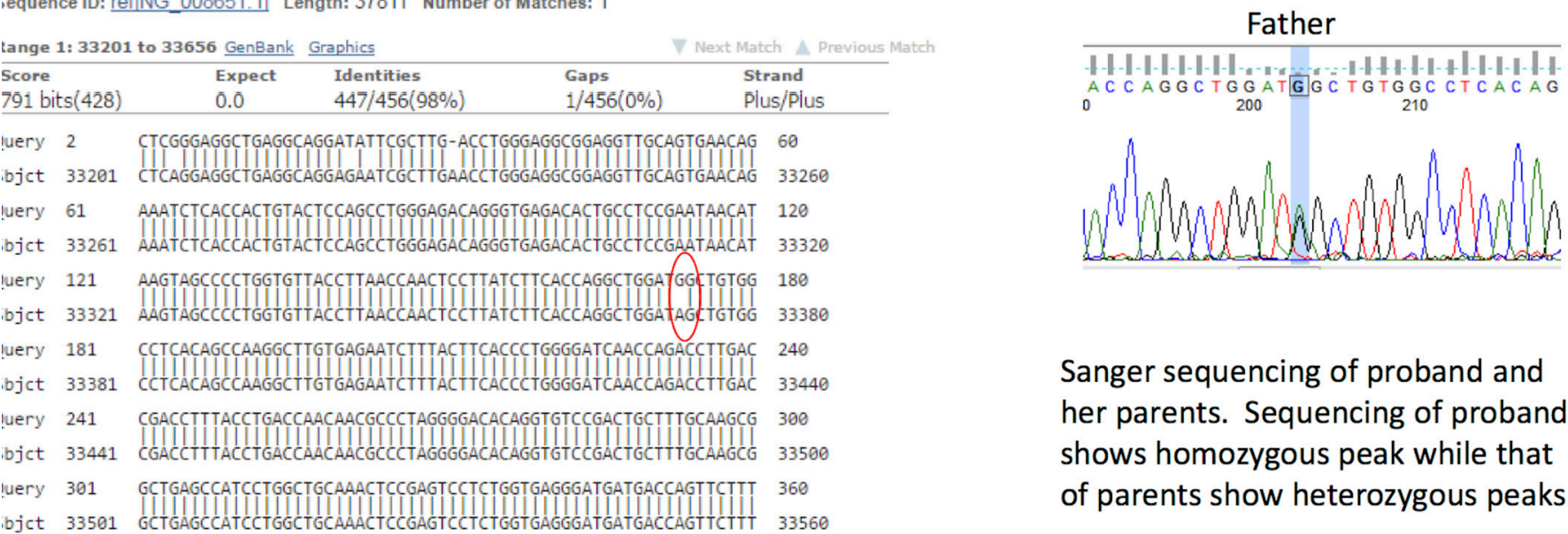

\begin{abstract}
Sanger sequencing of proband and her parents. Sequencing of proband shows homozygous peak while that of parents show heterozygous peaks.
\end{abstract}

Figure 1 Representative Sanger sequencing results of the patient compared with those of parents. NLR, nucleotide oligomerization domain-like receptor. 
There are still some caveats, such as homozygous variants are not known in this disorder nor have a mutation in LRR region been described. In view of these facts, the variant was classified as VOUS. These variants need to be tested in vitro to know their pathogenic significance. Widespread availability of whole exome sequencing is likely to identify many more such variants and in patients with atypical autoinflammatory syndrome, they may have significance.

\section{Latika Gupta, Sakir Ahmed, ${ }^{\ominus}$ Bharati Singh, Satya Prakash, Shubha Phadke, Amita Aggarwal}

Sanjay Gandhi Postgraduate Institute of Medical Sciences, Lucknow, India

Correspondence to Professor Amita Aggarwal, Sanjay Gandhi Post Graduate Institute of Medical Sciences, Lucknow 226014, India; aa.amita@gmail.com

Contributors The conception and design of the correspondence, acquisition of data, and analysis and interpretation of data: LG, SA, SPr, SPh and AA; Involved in care of the patient: $L G, B S, S P h$ and AA; Drafting the article: $L G$ and SA; Revising it critically for important intellectual content: BS, SPr, SPh and AA; Final approval of the version to be submitted: all authors; Agreement to be accountable for all aspects of the work in ensuring that questions related to the accuracy or integrity of any part of the work are appropriately investigated and resolved: all authors.

Funding The authors have not declared a specific grant for this research from any funding agency in the public, commercial or not-for-profit sectors.

Competing interests None declared.

Patient consent for publication Obtained.
Provenance and peer review Not commissioned; internally peer reviewed.

(c) Author(s) (or their employer(s)) 2019. No commercial re-use. See rights and permissions. Published by BMJ.

$$
\text { Check for updates }
$$

To cite Gupta L, Ahmed S, Singh B, et al. Ann Rheum Dis Epub ahead of print: [please include Day Month Year]. doi:10.1136/annrheumdis-2019-216158

Received 14 August 2019

Accepted 16 August 2019

Ann Rheum Dis 2019;0:1-2. doi:10.1136/annrheumdis-2019-216158

\section{REFERENCES}

1 Ter Haar NM, Eijkelboom C, Cantarini L, et al. Clinical characteristics and genetic analyses of 187 patients with undefined autoinflammatory diseases. Ann Rheum Dis 2019:pii: annrheumdis-2018-214472.

2 Tuncer S, Fiorillo MT, Sorrentino R. The multifaceted nature of NLRP12. J Leukoc Biol 2014:96:991-1000

3 Vitale A, Rigante D, Maggio MC, et al. Rare NLRP12 variants associated with the NLRP12-autoinflammatory disorder phenotype: an Italian case series. Clin Exp Rheumatol 2013;31(3 Suppl 77):155-6.

4 Kostik MM, Suspitsin EN, Guseva MN, et al. Multigene sequencing reveals heterogeneity of NLRP12-related autoinflammatory disorders. Rheumatol Int 2018:38:887-93.

5 Shen M, Tang L, Shi X, et al. NLRP12 autoinflammatory disease: a Chinese case series and literature review. Clin Rheumatol 2017:36:1661-7. 